\title{
Relativistic electron dropouts by pitch angle scattering in the geomagnetic tail
}

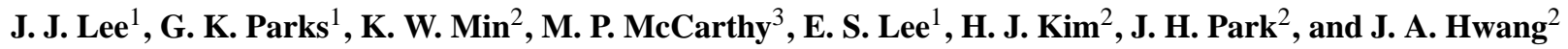 \\ ${ }^{1}$ Space Sciences Lab., University of California, Berkeley, CA, USA \\ ${ }^{2}$ Department of Physics, Korea Advanced Institute of Science and Technology, Daejeon, Korea \\ ${ }^{3}$ Geophysics Program, University of Washington, Seattle, USA
}

Received: 5 June 2006 - Revised: 22 August 2006 - Accepted: 13 October 2006 - Published: 22 November 2006

\begin{abstract}
Relativistic electron dropout (RED) events are characterized by fast electron flux decrease at the geostationary orbit. It is known that the main loss process is non adiabatic and more effective for the high energy particles. RED events generally start to occur at midnight sector and propagate to noon sector and are correlated with magnetic field stretching. In this paper, we discuss this kind of event can be caused from pitch angle diffusion induced when the gyro radius of the electrons is comparable to the radius of curvature of the magnetic field and the magnetic moment is not conserved any more. While this process has been studied theoretically, the question is whether electron precipitation could be explained with this process for the real field configuration. This paper will show that this process can successfully explain the precipitation that occurred on 14 June 2004 observed by the low-altitude $(680 \mathrm{~km})$ polar orbiting Korean satellite, STSAT-1. In this precipitation event, the energy dispersion showed higher energy electron precipitation occurred at lower $\mathrm{L}$ values. This feature is a good indicator that precipitation was caused by the magnetic moment scattering in the geomagnetic tail. This interpretation is supported by the geosynchronous satellite GOES observations that showed significant magnetic field distortion occurred on the night side accompanying the electron flux depletion. Tsyganenko01 model also shows the magnetic moment scattering could occur under the geomagnetic conditions existing at that time. We suggest the pitch angle scattering by field curvature violating the first adiabatic invariant as a possible candidate for loss mechanism of relativistic electrons in radiation belt.
\end{abstract}

Keywords. Magnetospheric physics (Energetic particles, precipitating; Energetic particles, trapped; Magnetotail)

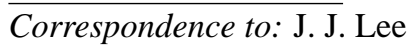

(jjlee@ssl.berkeley.edu)

\section{Introduction}

Many spacecraft failures and anomalies have been attributed to relativistic electrons (sometimes referred to as "killer electrons") in the Earth's magnetosphere (Baker, 2000). An example of such failures is caused by the spacecraft internal charging (Garrett and Whittlesey, 2000). High energy electrons can penetrate the thick shielding and bury themselves in the insulation around sensitive satellite electronics. Charges from these accumulating electrons build up in the spacecraft, and a strong internal electrical discharge can occur to destroy electric devices. Not surprisingly, understanding them has been one of top issues since early space era.

While the dynamics of these electrons have been studied extensively for several decades, the fundamental questions as to how they are produced and lost are still not fully resolved (Friedel et al., 2002). Electrons within Earth's magnetosphere come from two main sources, the solar wind and the Earth's ionosphere. However, these two sources provide low energy electrons, $1 \mathrm{eV}$ and $10 \mathrm{eV}$ for each. These values are too small to explain the high population of relativistic electrons with energies $\sim 100 \mathrm{keV}$ to $>15 \mathrm{MeV}$ ( $\mathrm{Li}$ and Temerin, 2001).

Regarding the question of how they are lost, there are two kinds of loss processes, adiabatic and non-adiabatic. The adiabatic processes, such as the magnetopause shadowing and $D_{s t}$ effect, do not represent real losses, because the flux decrease is caused by magnetic field intensity changes (Dessler and Karplus, 1961; Reeves et al., 1998; Kim and Chan, 1997). When the field returns, the flux will be recovered in the adiabatic process. Non-adiabatic processes are irreversible particle losses, where particles are lost permanently when they encounter the magnetopause(Desorgher et al., 2000) or precipitate into atmosphere by wave particle interactions or magnetic moment scattering due to field stretching(Young et al., 2002).

Published by Copernicus GmbH on behalf of the European Geosciences Union. 


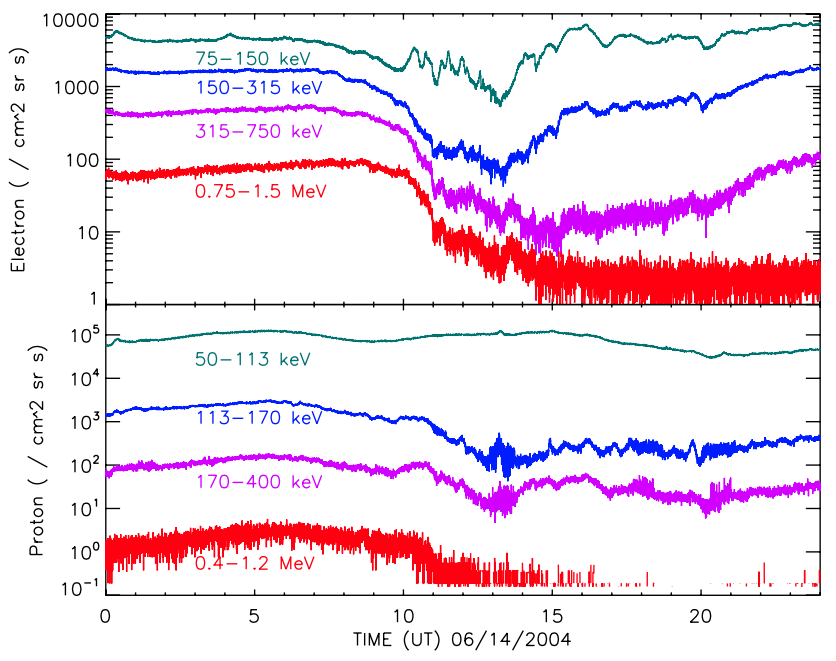

Fig. 1. Electron (top) and proton (bottom) flux observed by LANL02A on 14 June 2004.

The electron flux level in the magnetosphere at any time is determined by a competition between acceleration and loss process. The electron fluxes are commonly observed to decrease during the main phase of a storm, and then may increase to well above the prestorm levels during or shortly after the recovery phase of the storm(Nagai, 1988; Kim et al., 2006). Yet in some cases, electron fluxes are not recovered and remained low flux level(Reeves et al., 2003). Onsager et al. (2002) investigated the flux level at geosynchronous altitudes in response to a moderate $\left(-80 \mathrm{nT} D_{s t}\right)$ magnetic storm (16 April 2000). They found that the $>2 \mathrm{MeV}$ electrons decrease fairly abruptly but not simultaneously at different local times and the flux level did not increase during the recovery time. These events are known as RED (Relativistic Electron Dropout) events. They also showed that the lower energy electrons $<300 \mathrm{keV}$ recover fully while the $>2 \mathrm{MeV}$ electrons are permanently lost.

Green et al. (2004) studied three possible causes of the ensuing flux decrease: adiabatic electron motion in response to the changing magnetic flux topology, drift of the guiding center out the magnetopause boundary, and precipitation into the atmosphere. Although the adiabatic motion may be a satisfactory explanation for the local time dependence of the flux levels and partly contributed to electron flux decrease, their analysis revealed that electron flux dropouts are likely due to enhanced precipitation into the atmosphere. These flux dropouts showed a good correlation with the stretching of the magnetic field to a more tail-like configuration. Recently, Clilverd et al. (2006) observed large-scale precipitation into the atmosphere during the sudden decrease of geosynchronous electron flux $(>2 \mathrm{MeV})$. However, their indirect observation of precipitation with ground-based ionospheric measurements doesn't show clearly the correlation between RED events and electron precipitations. In this paper, we use in-situ data to demonstrate a RED event can cause particle precipitation.

It has been known that the increased field line curvature on stretched field lines could lead to the breaking of the first adiabatic invariant which can lead to precipitation of particles. Proton isotropic boundary might be explained by the proton precipitation due to magnetic moment scattering (Sergeev and Gvozdevsky, 1995). Friedel et al. (2002) stated "While this certainly can occur for protons, can this be a process for highly relativistic electrons also? Research in this area is ongoing." While the idea that magnetic moment scattering can lead to particle precipitation has been studied theoretically (Delcourt and Martin, 1994; Buechner and Zelenyi, 1989; Chen and Palmadesso, 1986), observational analysis has not made much progress. This is because it is difficult to identify the various sources of precipitation. Imhof et al. (1977) observed energy dispersion of precipitating electrons at the trapping boundary and interpreted that these events might occur from the violation of the first adiabatic invariant (Imhof et al., 1979, 1997; Imhof, 1988). Popielawska and Zwolakowska (1991) used Tsyganenko-87 and -89 model to reproduce the observed sharp decrease of threshold energy with increasing L. While their results show some agreements with measurements in latitude where isotropic precipitation was expected to occur, the very steep profiles of energy dispersion were not well explained. This paper will show the energy dispersion structures can indeed be reproduced with Tsyganenko-01 model using energetic electron data measured by STSAT-1. We estimate how fast electrons can be lost with a simple test particle simulation. Our results support the idea that this process can produce relativistic electron dropouts at the geostationary orbit and can help in the development of a comprehensive model of relativistic electron dynamics to forecast space weather.

\section{Relativistic electron dropouts}

An example of relativistic electron dropouts shown in the top panel of Fig. 1 was observed by the geostationary satellite, LANL-02A on 14 June 2004 in the energy range from $75 \mathrm{keV}$ to $1.5 \mathrm{MeV}$. Note that the $0.75 \sim 1.5 \mathrm{MeV}$ electron flux decreases were observed for $\sim 5 \mathrm{~h}$ at a level that is less than one tenth of the previous flux, while the lower energy $(\sim 100 \mathrm{keV})$ electron flux recovered after $\sim 1 \mathrm{~h}$. The flux drop out remained until 15 June and then slowly recovered to the previous level. As shown in the bottom panel of Fig. 1, the proton flux also decreased concurrent with the electrons. This figure demonstrates that the loss process is more effective for the higher energy electrons and protons.

The local-time progression of the flux dropout at geostationary orbit is shown in Fig. 2 with seven satellites, five LANL satellites and two GOES satellites. In this figure, closed and open circles indicate high and low electron flux 

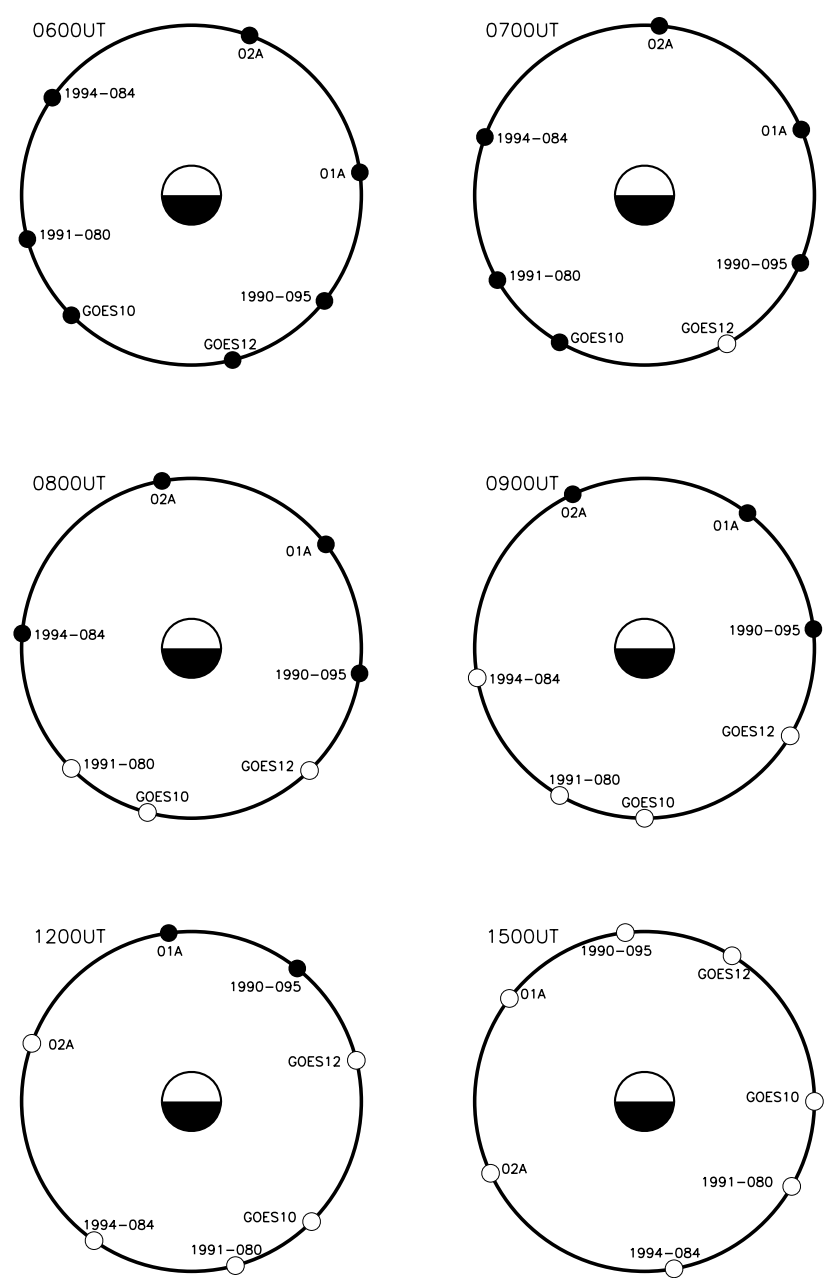

Fig. 2. Local time progression of electron flux dropouts observed by seven geosynchronous spacecraft. Black circles indicate high level flux, while open circles shows electron flux dropouts.

levels, respectively. All satellites observed high electron flux at 06:00 UT, but GOES12 first observed electron dropout between 06:00 and 07:00 UT. This dropout was fairly localized as can be seen by the fact that two satellites, GOES 10 and 1990-095 which were separated by $4 \mathrm{~h}$ and $3 \mathrm{~h}$ in local time from GOES12, didn't see the flux dropout. Somewhat later, electron dropout started to be detected by other satellites and propagated to noon sector from midnight. This result is a little different from Onsager et al. (2002) in which the electron dropout was observed first at dusk (15:00 LT). However, these observations are consistent with the statistical results of Green et al. (2004) who analyzed statistically 52 flux decreases and showed the electron flux is reduced first in the dusk sector (15:00 LT 24:00 LT) concurrent with the stretching of the magnetic field. Also their superposed epoch plot showed electron depletion started most frequently around 22:00 LT and propagated to all local times after $8 \mathrm{~h}$.

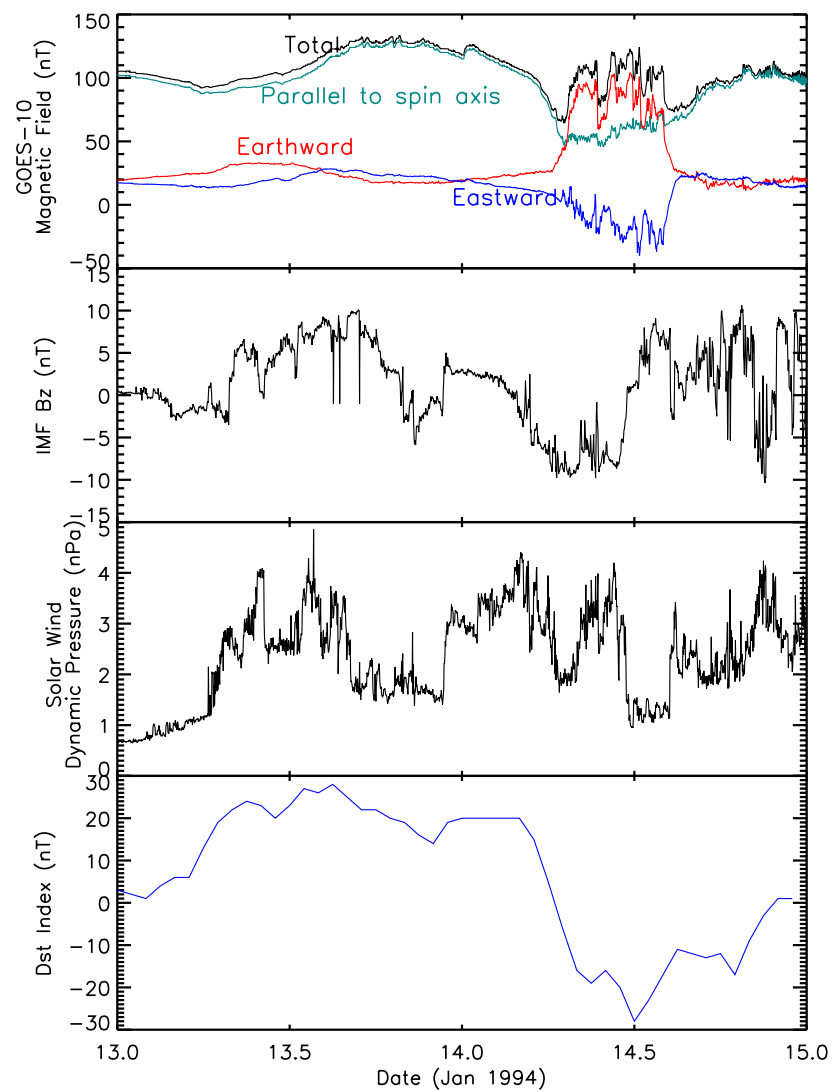

Fig. 3. Geomagnetic field observed by GOES-10(top), IMF Bz (second) and solar wind pressure measured by WIND and $D_{s t}$ index from 13 to 14 June 2004.

The time required for the dropout to be detected at all local times $(\sim 8 \mathrm{~h})$ was much longer than 5 to 10 -min drift period of the electrons. This implies the dropout did not occur through the depletion of the drift shells, but rather due to the distortion of the drift shells (Onsager et al., 2002).

Green et al. (2004) showed electron dropout is correlated with geomagnetic field stretching. The top panel of Fig. 3 shows the geomagnetic field measured by the geostationary satellite, GOES-10, from 13 to 14 June. The red line indicates earthward direction and green line shows parallel direction to the satellite spin axis, approximately $+Z$ in GSM coordinate. Comparing to the magnetic field data observed on 13 June, the earthward magnetic field was much enhanced while $+Z$ direction field strength decreased at 12:00 UT on 14 June. Because GOES-10 passed through the midnight sector at 09:20 UT, this field data imply the geomagnetic field was stretched to more tail-like geometry at the night time. Superposed epoch analysis performed by Green et al. (2004) shows electron dropout events are observed preceding quiet condition, occurs with solar wind pressure enhancement, southward turning of $B_{z}$, decrease of $D_{s t}$ and increase of AE indices. During the event, from 06:00 UT to 15:00 UT 


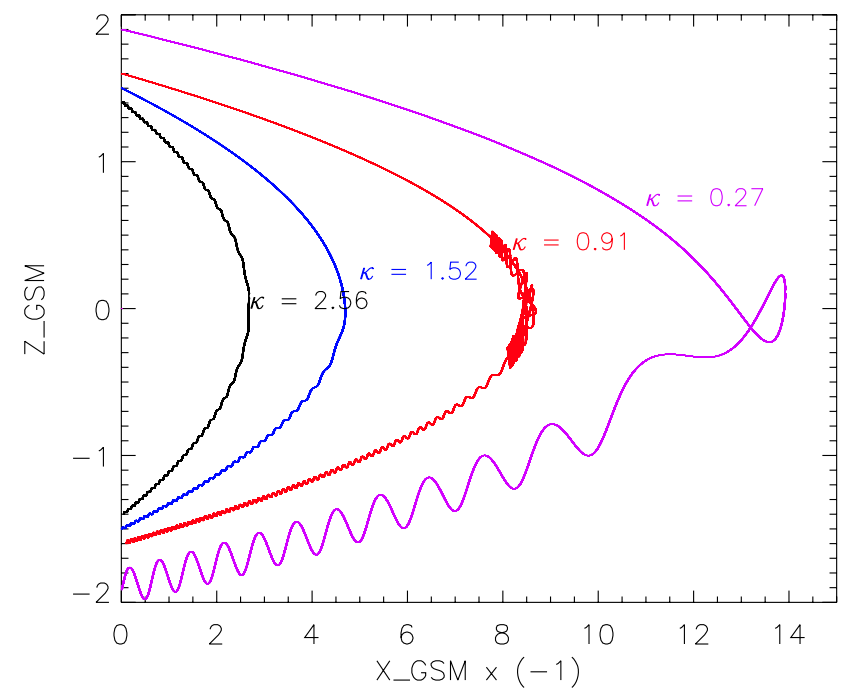

Fig. 4. Electron trajectories for field lines having four different $\kappa$, 2.56 (black), 1.52 (blue), 0.91 (red) and 0.27 (purple).

on 14 June, WIND measured strong southward IMF Bz, almost $-10 \mathrm{nT}$, and enhanced solar wind pressure as shown in the second and third panels of Fig. 3. The bottom panel of the Fig. 3 shows the $D_{s t}$ index decreased from $15 \mathrm{nT}$ to $-30 \mathrm{nT}$. These variations of geophysical indices however do not explain how the dramatic change of magnetic fields are produced. Nor is it clear how these variations can produce field line stretching. The following summarizes the electron dropout characteristics.

1. Fast dropouts last for $1 \sim 5 \mathrm{~h}$

2. Observed for both of electrons and protons

3. More effective for higher energy charged particles

4. Started from dusk and midnight sector and propagate to noon sector

5. Correlation with magnetic field stretching

\section{Pitch angle scattering by field curvature}

The violation of the first adiabatic invariant has strict constraint for electrons in the magnetosphere because it requires perturbation with time scales of one gyro period. However, the first adiabatic invariant can be violated if the radius of magnetic field curvature is comparable to the electron gyro radius (Chen and Palmadesso, 1986; Buechner and Zelenyi, 1989; Delcourt and Belmont, 1998). This is because during one gyro motion, an electron experiences significant magnetic field changes that cause pitch-angle changes so particles enter the loss cone. This magnetic moment scattering is characterized by $\kappa=\left[R_{c} / \rho\right]^{1 / 2}$, where $R_{c}$ is the minimum radius of magnetic field curvature and $\rho$ is the particle's maximum gyro radius. It's not easy to define clearly the $\kappa$ values for real magnetic field, because the field has three dimensional configuration and depends on location. For a parabolic field model, $\mathbf{B}(z)=b_{x} \frac{z}{L} \hat{x}+b_{z} \hat{z}$, we define $\kappa$ as above, evaluated for a charged particle at $z=0$ where the radius of curvature is minimum. In this case, the radius of curvature is $L b_{z} / b_{x}$ and the gyro radius is $p /\left(q b_{z}\right)$ with $p$ and $q$ the particle's momentum and charge (Delcourt and Belmont, 1998). Figure 4 shows how the electron trajectories depend on $\kappa$ for four different values. The electron trajectories were calculated by solving the Lorentz equation for a parabolic field model, and electrons were launched from south to north with $30^{\circ}$ initial pitch angle. For three $\kappa$ values, 2.56 (black), 1.52 (blue) and 0.91 (red), $1 \mathrm{MeV}$ test electrons were used and for the $\kappa$ value of 0.27 (purple), we used $15 \mathrm{MeV}$ electrons.

The results indicate that for the line(black) having the largest radius of curvature, the terminal pitch angle is exactly same as the initial pitch angle. Here a $\kappa$ value of 2.56 is slightly larger than the value needed for electrons to undergo non-adiabatic motion. For the field (blue line) having smaller radius of curvature, electrons start to change their pitch angles during the passage through the field reversal region. The final pitch angle can be larger or smaller than initial pitch angle. And some electrons can have very small pitch angles, aligned along the field lines as shown in Fig. 4. For a detailed description about pitch angle scattering, see Delccourt's papers (Delcourt et al., 1996, 2004; Delcourt and Belmont, 1998; Delcourt and Martin, 1999).

As the radius of field line curvature decreases, the final pitch angle range that electrons can have increases. When $\kappa$ is 0.91 , some electrons having $30^{\circ}$ initial pitch angle can have $90^{\circ}$ pitch angle near the equator and move back and forth as chaotic motion (red line). Finally these electrons can be aligned to the magnetic field then precipitate into the atmosphere. For the smallest radius of curvature in Fig. 4, the electron motion (purple line), $\kappa$ is 0.27 . If $\kappa$ is much smaller than unity, particles may experience meandering motion about the field minimum as shown by Speiser (1965). Because electrons have small gyro radius, in the real magnetosphere field line, it is hard for electrons to follow the motions described by red or purple lines. We assumed electrons can be lost by only the pitch angle scattering described by blue line in Fig. 4.

To estimate how fast electrons can escape from the magnetosphere, we simulated the pitch angle distribution for a field line having $\kappa$ of 2.1 with 720000 test particles. For this $\kappa$, electrons can change their pitch angles within $1.0^{\circ}$. Here we assumed the loss cone is just $0.7^{\circ}$ whose value is based on the magnetic field derived from Tsyganenko-01 model (Tsyganenko, 2002) where the magnetic field is $52940 \mathrm{nT}$ at $100 \mathrm{~km}$ altitude and the equatorial field is $7.9 \mathrm{nT}$. We thus considered test electrons having pitch angles from $0.7^{\circ}$ to $5^{\circ}$ as shown in the top panel of Fig. 5 where the angles are calculated for the equatorial magnetic field assuming the 
magnetic moment is conserved. The bottom panel of Fig. 5 shows how the pitch angle distribution changed after just one pass through the equator. Note the electron flux in the loss cone increased to almost the same level as outside of the loss cone, which means the loss cone was filled by a strong pitch angle scattering process. With this process, $0.0075 \%$ electrons should be lost by precipitation for half bouncing motion. Here we assumed pitch angle distribution is uniform at the outside of loss cone. Because only electrons within $1^{\circ}$ from the loss cone can diffuse into loss cone, loss rate depends on how many times they pass through equatorial region where magnetic moment scattering occurs. The diffusion of electrons away from the loss cone contributes to keeping uniform pitch angle distribution and supplies electrons to the region near loss cone. The loss rate can be calculated from the equation, $d F / d t=(-2 \times 0.000075) F / T_{B}$, where $\mathrm{F}$ is electron flux and $T_{B}$ is a bouncing period. The time taken to reduce the electron flux from the equatorial region of the radiation belt by a factor of $1 / \mathrm{e}$ is $T_{B} /(2 \times 0.000075)$. Assuming $1 \mathrm{MeV}$ electrons travel $20 \mathrm{Re}$ for one bouncing motion, $T_{B}$ is about $425 \mathrm{~ms}$. Assuming electron precipitation occurs in the region of $90^{\circ}$ in longitude ( $25 \%$ of a drift circle), our rough calculation shows it takes just $3.1 \mathrm{~h}$ to empty radiation belts.

\section{Observations of electron precipitation}

The pitch angle scattering by the field line curvature can explain many features of electron dropouts. First, we calculated it takes about $3.1 \mathrm{~h}$ to empty an electron flux tube from the radiation belt. This can explain reasonably well the geostationary spacecraft observations of large and fast flux dropouts that takes several hours. The magnetic moment scattering process by field line curvature is more effective for high energy electrons, which can explain why low energy electron dropout is not observed. The magnetic field curvature scattering is most important in the region where field curvature radius is minimum which might correspond to midnight sector and far away from the earth. Thus large L shell electrons can escape more easily. This can explain the observations that electron dropouts begin near midnight and propagate to noon sector. Some other observations, Onsager et al. (2002) in which electron dropouts start from dusk side can be explained by the formation of a partial ring current driven by changing solar wind conditions (Green et al., 2004). Moreover, this process is effective for both protons and electrons. If $1 \mathrm{MeV}$ electrons had $\kappa$ of $1.52,1 \mathrm{MeV}$ proton would have $\kappa$ of 0.27 for the same field configuration. Thus, the same energy electrons and protons undergo very different motions. Nevertheless, both of them have a chance to precipitate into the Earth's atmosphere by pitch angle scattering if $\kappa$ is smaller than the critical value of about 2.5 as shown in Fig. 4. Yet significant proton flux drop is not commonly observed in the low energy region (Fig. 1).
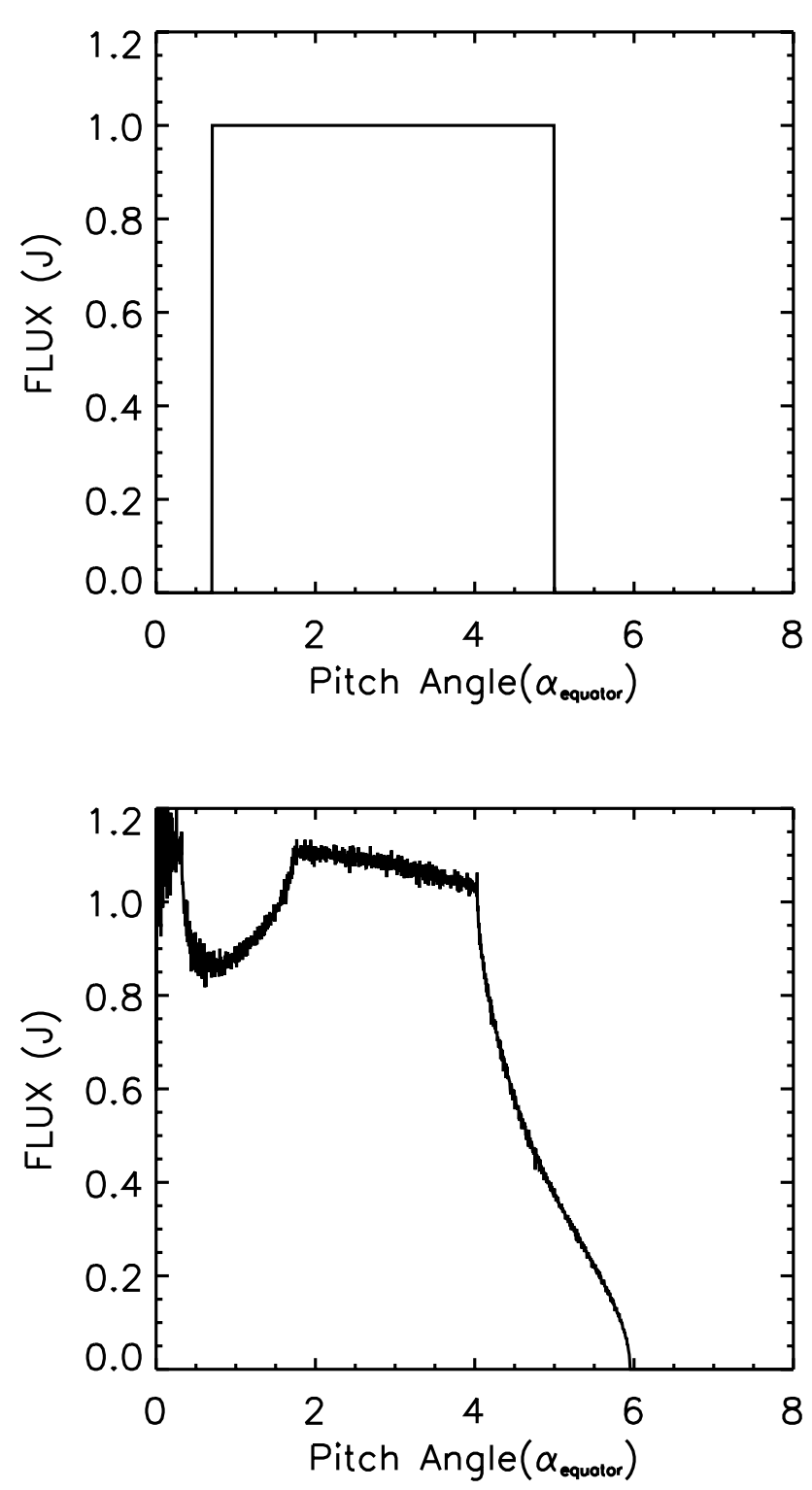

Fig. 5. Loss cone filling by pitch angle scattering. Initial pitch angle distribution (top), changed pitch angle distribution after passing through field reversal (bottom).

This is possibly due to the fact that the particle's loss rate depends on the number passing through the field reversal region. The gyro radius of $100 \mathrm{keV}$ protons is ten times larger than $1 \mathrm{MeV}$ electrons, but the velocity is just $1.5 \%$ of electrons. This means that it takes longer time to deplete protons as electrons.

Though magnetic moment scattering process can explain many features about relativistic electron dropouts, can we say with certainty that the magnetic moment scattering mechanism was really responsible on 14 June? To test this theory, we show in Fig. 6 an electron spectrogram obtained by 

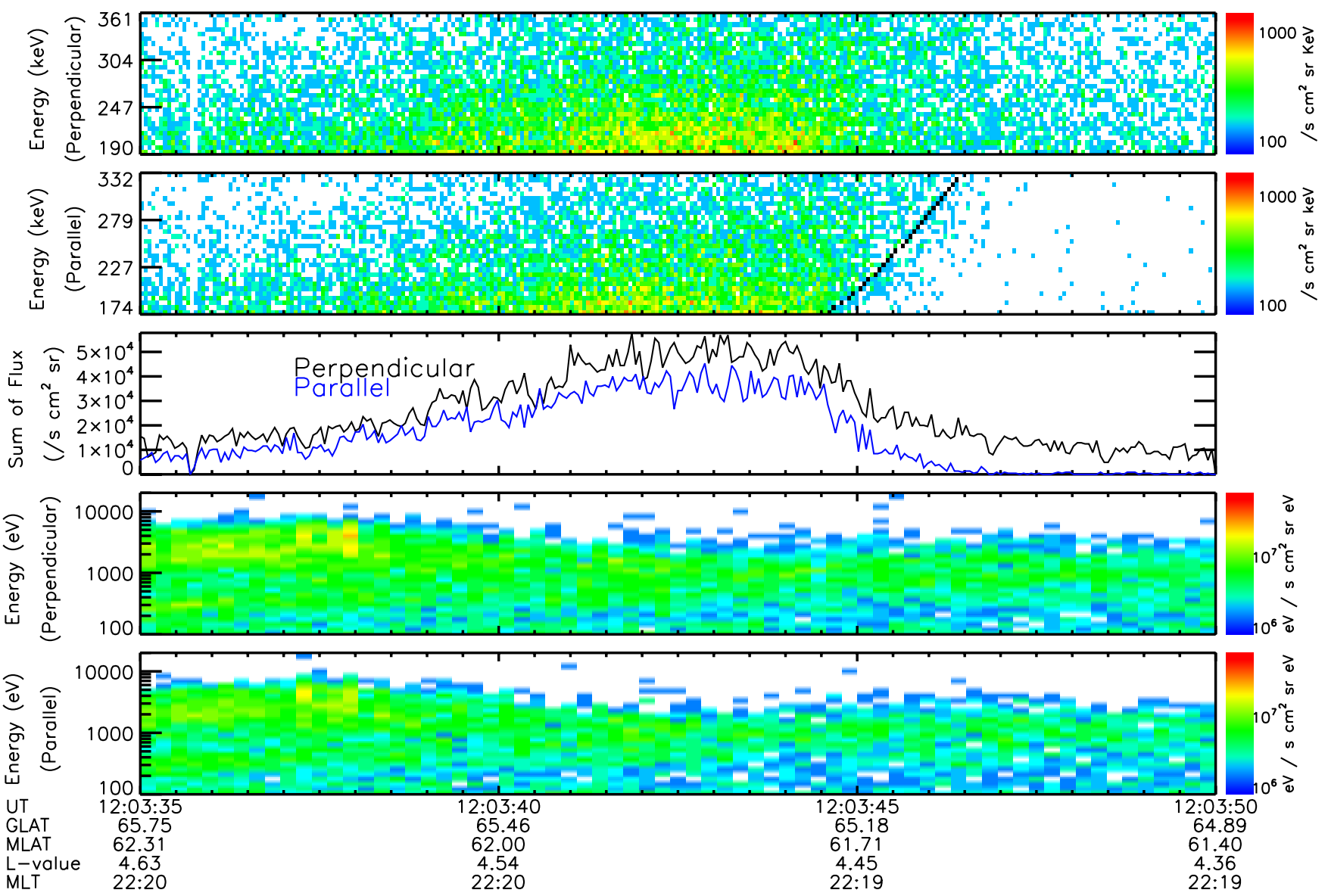

Fig. 6. Energetic electron precipitations observed by STSAT-1 on 14 June 2004. The black line in the second panel indicates energy dispersion calculated in Fig. 7.

STSAT-1. STSAT-1 was launched on 27 September 2003 into a sun synchronous low altitude $(680 \mathrm{~km})$ orbit. STSAT1 instrumentation included the Far-ultraviolet Imaging Spectrograph (FIMS), an Electro-Static Analyzer (ESA), Solid State Telescopes (SST), a Langmuir Probe (LP) and Scientific Magnetometer (SM). FIMS is an astrophysical instrument while the latter four are space physics experiment. These four instruments operate simultaneously in the polar region.

SST has two solid state detectors to measure energy spectra of electrons between $170 \mathrm{keV} \sim 360 \mathrm{keV}$. Each detector has a field of view of $33.9^{\circ}$, one is parallel and the other is perpendicular to the Earth magnetic field. Because the atmospheric loss cone at $680 \mathrm{~km}$ is about $60^{\circ}$, the perpendicular SST detects trapped electrons mirroring at the satellite altitude while the parallel SST which looks upward detect precipitated electrons. The top two panels show differential energy spectra from the perpendicular and parallel SSTs. The third panel shows the total electron fluxes in the energy measured by the SSTs in the two directions. For comparison with the SST electrons, the perpendicular and parallel components of energy flux spectrograms measured by Electrostatic Analyzer (ESA) are shown in the bottom two panels. A detailed description of the instruments is in Lee et al. (2005).

As shown in the second panel, strong electron precipitation was observed while significant precipitation was not observed in the low energy spectrogram. With excellent time resolution of SST, $50 \mathrm{~ms}$, an energy dispersion structure whose width is about $0.12^{\circ}$ was resolved at the lower latitude boundary of electron precipitation. Such energy dispersion structure was reported first by Imhof et al. (1977). They interpreted the energy dispersion structure as resulting from magnetic moment scattering. Because the larger radius of magnetic field curvature generally corresponds to the lower latitude, only high energy electrons can be precipitated at the low latitude. Thus, if we measured electron precipitation generated by magnetic moment scattering, energy dispersion structure should be observed at the precipitation boundary. 


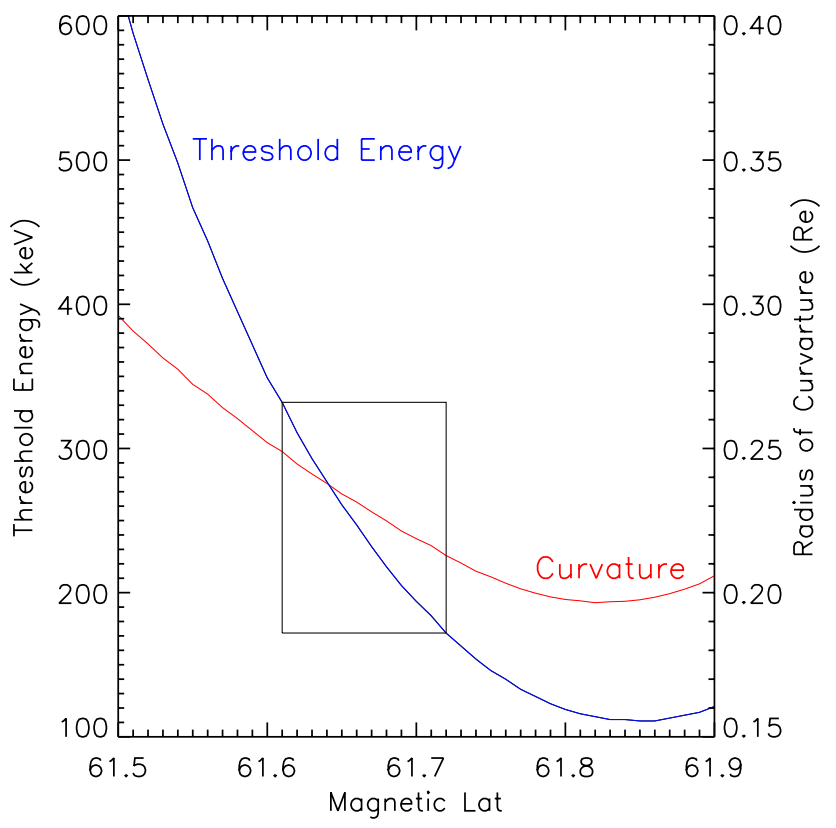

Fig. 7. The radius of magnetic field curvature and threshold energy derived from Tsyganenko01 model.

In Fig. 7, the magnetic field curvatures and threshold energies are plotted for the magnetic latitude of interest. The threshold energy is defined by the energy corresponding to $\kappa$ of 2.5 which can induce electron loss by pitch angle scattering. The rectangular box shows the energy range measured by STSAT-1 parallel SST, $170 \mathrm{keV} \sim 330 \mathrm{keV}$ and the corresponding threshold energy. We derived approximately the radius of curvature from Tsyganenko-01 model where the input parameters used were $D_{s t}$ index of $-33 \mathrm{nT}$, solar wind pressure of $2.81 \mathrm{nPa}$, IMF By of $7.25 \mathrm{nT}$ and $\mathrm{Bz}$ of $-9.10 \mathrm{nT}$. These values are slightly different from real measurements where $D_{s t}$ index was $-20 \mathrm{nT}$ and IMF Bz was $-7.1 \mathrm{nT}$. These solar wind and IMF data were measured by WIND at 11:00 UT, while $D_{s t}$ were measured at 12:00 UT. The time difference of $1 \mathrm{~h}$ is the time for solar wind whose velocity is $400 \mathrm{~km} / \mathrm{s}$ to reach the Earth from the WIND spacecraft which was located at $-214 \mathrm{Re}$ along X (GSE coordinate). Actually we had some difficulties in selecting these parameters, because there were fluctuations (Fig. 3), and ACE and WIND showed somewhat different data. Considering Tsyganenko model is a statistical model, we think these changes of IMF $\mathrm{Bz}$ and $D_{s t}$ index are reasonably applicable in modeling the magnetic field on 14 June. Figure 7 reproduces extremely well the energy dispersion observed by STSAT- 1 where the dispersion appeared from $61.72^{\circ}$ to $61.84^{\circ}$ in geomagnetic latitude, which is the same as the result obtained from Tsyganenko model. We showed this dispersion in Fig. 6 with black line. This result strongly suggests that electrons were precipitated on 14 June by the field curvature magnetic moment scattering.

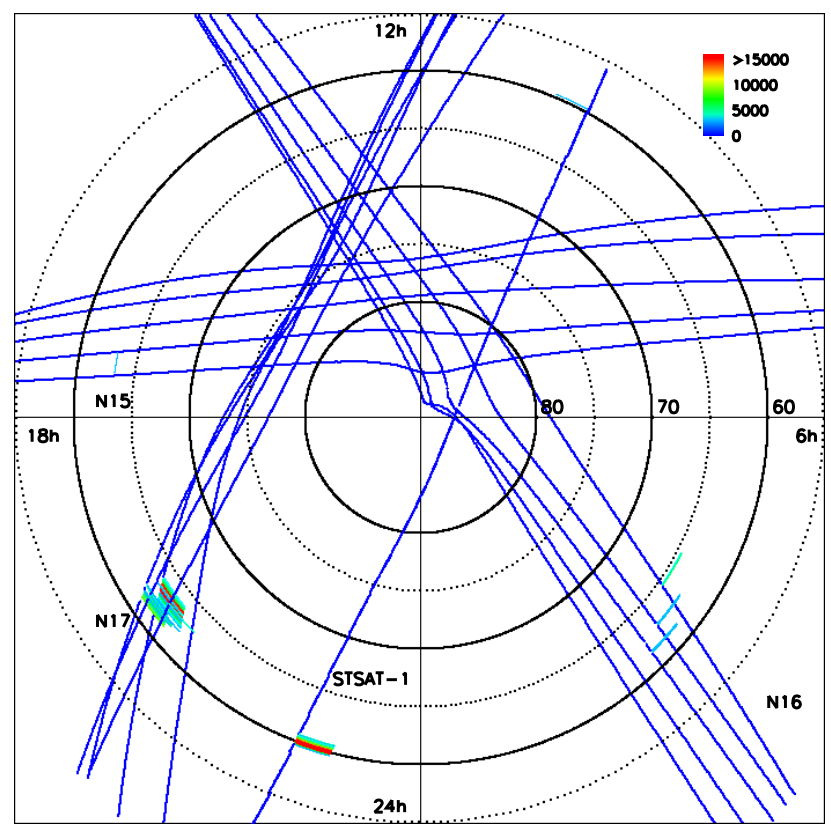

Fig. 8. Electron precipitations observed by four spacecraft, NOAA/POES N15, N16, N17 and STSAT-1.

Our results strongly support the idea that pitch angle scattering by curvature contributes to the electron flux dropouts. However, we do not exclude other precipitation mechanisms, such as a non-adiabatic loss process, e.g. precipitation by wave and particle interactions. It has been known that electrons in the magnetosphere can interact with electron cyclotron harmonic waves (ECH) (Horne and Thorne, 2000), electromagnetic ion cyclotron waves (EMIC) (Summers and Thorne, 2003; Albert, 2003; Lorentzen et al., 2000; Horne and Thorne, 1998), and whistler waves (Lorentzen and Blake, 2001). These waves are generally observed in different local time sectors. ECH waves are predominantly seen in the midnight to dawn region of magnetosphere associated with substorm injections. EMIC waves occur in the dusk region where the ratio between the plasma frequency and electron gyro frequency is high, and whistler waves are observed in the dawn sector.

Figure 8 shows where energetic electron precipitations were observed by STSAT-1 and three NOAA/POES satellites, N15, N16 and N17. The blue line indicates satellite trajectories from 06:00 UT to 15:00 UT in geomagnetic coordinates. STSAT-1 observation was made just in one orbit, while NOAA/POES made five orbit observations. NOAA/POES is at low altitude, $830 \mathrm{~km}$, and polar orbiting. Only NOAA/POES N17 detected significant irregular precipitation signature which energy is $>300 \mathrm{keV}$ near 21:00 LT. It's not clear whether this is related with the precipitation observed by STSAT-1 around 23:14 MLT. Yet this figure implies that the relativistic electron dropout event on 
14 June is unlikely to be caused by EMIC or whistler mode waves, because these waves are confined to the dusk and dawn regions. At the present time, there are no wave particle interaction theories that can generate the energy dependent electron flux dropouts as seen by STSAT- 1 .

\section{Conclusions}

In this paper, we showed precipitation produced by field curvature can explain well the characteristics of relativistic electron dropout events observed on 14 June 2004. While this event showed a slow recovery, it is important to note that electron dropout events are usually accompanied with sudden electron enhancements. Thus we need to understand acceleration and loss mechanisms to develop a model of relativistic electron dynamics to forecast space weather. In conclusion, the pitch angle scattering induced by field curvature should be viewed as a serious candidate for loss mechanisms of relativistic electrons in the radiation belt.

Acknowledgements. This work is supported by a NASA grant NAG5-11416. We would like to thank all engineers at SaTReC for contributing to successful operation of scientific mission of STSAT1.

Topical Editor I. A. Daglis thanks S. Bourdarie and another referee for their help in evaluating this paper.

\section{References}

Albert, J. M.: Evaluation of quasi-linear diffusion coefficients for EMIC waves in a multispecies plasma, J. Geophys. Res., 108, 16-1, doi:10.1029/2002JA009792, 2003.

Baker, D.: The occurrence of operational anomalies in spacecraft and their relationship to space weather, IEEE Trans. Plasma Sci, 28, 2007-2016, 2000.

Buechner, J. and Zelenyi, L. M.: Regular and chaotic charged particle motion in magnetotaillike field reversals. I - Basic theory of trapped motion, J. Geophys. Res., 94, 11 821-11 842, 1989.

Chen, J. and Palmadesso, P. J.: Chaos and nonlinear dynamics of single-particle orbits in a magnetotaillike magnetic field, J. Geophys. Res., 91, 1499-1508, 1986.

Clilverd, M. A., Rodger, C. J., and Ulich, T.: The importance of atmospheric precipitation in storm-time relativistic electron flux drop outs, Geophys. Res. Lett., 33, L1102, doi:10.1029/ 2005GL024661, 2006.

Delcourt, D. C. and Belmont, G.: Particle Dynamics in the NearEarth Magnetotail and Macroscopic Consequences, in: New Perspectives on the Earth's Magnetotail, edited by: Nishida, A., Baker, D. N., and Cowley, S. W. H., pp. 193-210, 1998.

Delcourt, D. C. and Martin, R. F.: Application of the centrifugal impulse model to particle motion in the near-Earth magnetotail, J. Geophys. Res., 99, 23 583-23 590, 1994.

Delcourt, D. C. and Martin, R. F.: Pitch angle scattering near energy resonances in the geomagnetic tail, J. Geophys. Res., 104, 383394, doi:10.1029/1998JA900041, 1999.

Delcourt, D. C., Sauvaud, J.-A., Martin, R. F., and Moore, T. E.: On the nonadiabatic precipitation of ions from the near-
Earth plasma sheet, J. Geophys. Res., 101, 17409-17418, doi:10.1029/96JA01006, 1996.

Delcourt, D. C., Malova, H. V., and Zelenyi, L. M.: Dynamics of charged particles in bifurcated current sheets: The $\kappa \sim 1$ regime, J. Geophys. Res., 109, A01 222, doi:10.1029/2003JA010167, 2004.

Desorgher, L., Bühler, P., Zehnder, A., and Flückiger, E. O.: Simulation of the outer radiation belt electron flux decrease during the March 26, 1995, magnetic storm, J. Geophys. Res., 105, $21211-$ 21 224, doi:10.1029/2000JA900060, 2000.

Dessler, A. J. and Karplus, R.: Some Effects of Diamagnetic Ring Currents on Van Allen Radiation, J. Geophys. Res., 66, 22892296, 1961.

Friedel, R. H. W., Reeves, G. D., and Obara, T.: Relativistic electron dynamics in the inner magnetosphere - a review, J. Atmos. Terr. Phys., 64, 265-282, 2002.

Garrett, H. B. and Whittlesey, A. C.: Spacecraft charging, An update, IEEE Trans. Plasma Sci, 28, 20 017-2028, 2000.

Green, J. C., Onsager, T. G., O’Brien, T. P., and Baker, D. N.: Testing loss mechanisms capable of rapidly depleting relativistic electron flux in the Earth's outer radiation belt, J. Geophys. Res., 109, A12 211, doi:10.1029/2004JA010579, 2004.

Horne, R. B. and Thorne, R. M.: Potential waves for relativistic electron scattering and stochastic acceleration during magnetic storms, Geophys. Res. Lett., 25, 3011-3014, doi:10.1029/98GL01002, 1998.

Horne, R. B. and Thorne, R. M.: Electron pitch angle diffusion by electrostatic electron cyclotron harmonic waves: The origin of pancake distributions, J. Geophys. Res., 105, 5391-5402, doi:10.1029/1999JA900447, 2000.

Imhof, W. L.: Fine resolution measurements of the L-dependent energy threshold for isotropy at the trapping boundary, J. Geophys. Res., 93, 9743-9752, 1988.

Imhof, W. L., Reagan, J. B., and Gaines, E. E.: Fine-scale spatial structure in the pitch angle distributions of energetic particles near the midnight trapping boundary, J. Geophys. Res., 82, 5215-5221, 1977.

Imhof, W. L., Reagan, J. B., and Gaines, E. E.: Studies of the sharply defined L dependent energy threshold for isotropy at the midnight trapping boundary, J. Geophys. Res., 84, 6371-6384, 1979.

Imhof, W. L., Chenette, D. L., Gaines, E. E., and Winningham, J. D.: Characteristics of electrons at the trapping boundary of the radiation belt, J. Geophys. Res., 102, 95-104, doi:10.1029/96JA02797, 1997.

Kim, H.-J. and Chan, A. A.: Fully adiabatic changes in storm time relativistic electron fluxes, J. Geophys. Res., 102, 22 107-22 116, doi:10.1029/97JA01814, 1997.

Kim, H.-J., Kim, K. C., Lee, D.-Y., and Rostoker, G.: Origin of geosynchronous relativistic electron events, J. Geophys. Res., 111, A03 208, doi:10.1029/2005JA011469, 2006.

Lee, J.-J., Parks, G. K., Min, K. W., Kim, H. J., Park, J., Hwang, J., McCarthy, M. P., Lee, E., Ryu, K. S., Lim, J. T., Sim, E. S., Lee, H. W., Kang, K. I., and Park, H. Y.: Energy spectra of $\sim 170$ $360 \mathrm{keV}$ electron microbursts measured by the Korean STSAT-1, Geophys. Res. Lett., 32, L13 106, doi:10.1029/2005GL022996, 2005.

Li, X. and Temerin, M. A.: The Electron Radiation Belt, Space Sci. Rev., 95, 569-580, 2001. 
Lorentzen, K. R. and Blake, J. B.: Observations of relativistic electron microbursts in association with VLF chorus, J. Geophys. Res., 106, 6017-6027, 2001.

Lorentzen, K. R., McCarthy, M. P., Parks, G. K., Foat, J. E., Millan, R. M., Smith, D. M., Lin, R. P., and Treilhou, J. P.: Precipitation of relativistic electrons by interaction with electromagnetic ion cyclotron waves, J. Geophys. Res., 105, 5381-5390, doi:10.1029/1999JA000283, 2000.

Nagai, T.: 'Space weather forecast' - Prediction of relativistic electron intensity at synchronous orbit, Geophys. Res. Lett., 15, 425428, 1988.

Onsager, T. G., Rostoker, G., Kim, H.-J., Reeves, G. D., Obara, T., Singer, H. J., and Smithtro, C.: Radiation belt electron flux dropouts: Local time, radial, and particle-energy dependence, J. Geophys. Res., 107, 21-1, doi:10.1029/2001JA000187, 2002.

Popielawska, B. and Zwolakowska, D.: An assessment of a magnetospheric model by tracing the energetic particle trapping boundary, Geophys. Res. Lett., 18, 1489-1492, 1991.

Reeves, G. D., Friedel, R. H. W., Belian, R. D., Meier, M. M., Henderson, M. G., Onsager, T., Singer, H. J., Baker, D. N., Li, X., and Blake, J. B.: The relativistic electron response at geosynchronous orbit during the January 1997 magnetic storm, J. Geophys. Res., 103, 17 559-17 570, doi:10.1029/97JA03236, 1998.
Reeves, G. D., McAdams, K. L., Friedel, R. H. W., and O'Brien, T. P.: Acceleration and loss of relativistic electrons during geomagnetic storms, Geophys. Res. Lett., 30, 36-1, doi:10.1029/2002GL016513, 2003.

Sergeev, V. A. and Gvozdevsky, B. B.: MT-index - a possible new index to characterize the magnetic configuration of magnetotail, Ann. Geophys., 13, 1093-1103, 1995, http://www.ann-geophys.net/13/1093/1995/.

Speiser, T. W.: Particle Trajectories in a Model Current Sheet, Based on the Open Model of the Magnetosphere, with Applications to Auroral Particles, J. Geophys. Res., 70, 1717-1728, 1965.

Summers, D. and Thorne, R. M.: Relativistic electron pitchangle scattering by electromagnetic ion cyclotron waves during geomagnetic storms, J. Geophys. Res., 108, 2-1, doi:10.1029/2002JA009489, 2003.

Tsyganenko, N. A.: A model of the near magnetosphere with a dawn-dusk asymmetry 2. Parameterization and fitting to observations, J. Geophys. Res., 107, 10-1, doi:10.1029/2001JA000220, 2002.

Young, S. L., Denton, R. E., Anderson, B. J., and Hudson, M. K.: Empirical model for $\mu$ scattering caused by field line curvature in a realistic magnetosphere, J. Geophys. Res., 107, 3-1, doi:10.1029/2000JA000294, 2002. 